\title{
Aeroelastic analysis of long-span bridges using time domain methods
}

\author{
J. Á. Jurado, A. León, S. Hernández \& F. Nieto \\ School of Civil Engineering, University of Coruña, Spain
}

\begin{abstract}
The current calculation power of computers permits the aeroelastic analysis of long-span bridges using time domain methods. These methods solve the differential equations set for the dynamic analysis by means of step by step integration, taking into account the fluid structure interaction of the wind aeroelastic phenomena. Working with time domain methods has advantages over more standard frequency domain methods. It is possible to more accurately study non-stationary periods associated with the initiation of the aeroelastic instabilities, and nonlinearities can also be analyzed more easily. The main difficulty with time domain methods is the modelling of wind forces, usually defined as frequency functions. The above-mentioned drawback is solved by using the so-called indicial functions that depend on time and they must be obtained from the classical flutter derivatives, which are functions of the frequency. A recent method, named band superposition, has been developed. The new concept is the decomposition of the wind forces in different frequency ranges, analyzing the forces of the lowest frequency band as quasi-steady, and the higher frequency bands with their classical expressions in terms of frequency. To analyze the low-frequency wind loads as quasi-steady is appropriate because the wind flow fits to the deck geometry for that case. The total response consists of the sum of the responses for each frequency band. This paper explains and compares all these methods and shows examples.
\end{abstract}

Keywords: aeroelasticity, long-span bridges, time domain analysis, flutter, buffeting. 


\section{Flutter derivatives and indicial functions}

Flutter phenomenon in cable supported bridges is mathematically modelled in a similar way as flutter of an aerodynamic profile in aeronautics. However, the geometry of the cross section deck is not usually aerodynamic. Therefore, the functions that relate to the degrees of freedom considered and the aeroelastic forces on the deck do not have analytical expressions. The most widely used solution for this difficulty is the Simiu and Scanlan [1] method, which defines functions called flutter derivatives that depend on the vibration frequency and the mean wind speed. These functions are obtained experimentally, working with a sectional model of the deck in wind tunnels. According to the sign criteria shown in Figure 1, the expression of aeroelastic drag, lift and moment $\mathbf{f}_{a}=\left\{D_{a}, L_{a}\right.$, $\left.M_{a}\right\}^{T}$, self-excited by the deck movements $\mathbf{u}=\{h, p, \alpha\}^{T}$ can be written in matrix form as

$$
\mathbf{f}_{a}=\mathbf{C}_{a} \dot{\mathbf{u}}+\mathbf{K}_{a} \mathbf{u},
$$

where

$$
\mathbf{C}_{a}=\frac{1}{2} \rho V K B l \cdot\left(\begin{array}{ccc}
P_{1}^{*} & P_{5}^{*} & B P_{2}^{*} \\
H_{5}^{*} & H_{1}^{*} & B H_{2}^{*} \\
B A_{5}^{*} & B A_{1}^{*} & B^{2} A_{2}^{*}
\end{array}\right) \quad \mathbf{K}_{a}=\frac{1}{2} \rho V^{2} K^{2} l \cdot\left(\begin{array}{ccc}
P_{4}^{*} & P_{6}^{*} & B P_{3}^{*} \\
H_{6}^{*} & H_{4}^{*} & B H_{3}^{*} \\
B A_{6}^{*} & B A_{4}^{*} & B^{2} A_{3}^{*}
\end{array}\right),
$$

where $B$ is the deck width, $\rho$ is the air density, $l$ is the dimension along the deck, $V$ is the mean wind speed, $K=B \omega / V$ is the reduced frequency with $\omega$ the frequency of the response and $H_{i}^{*}, P_{i}^{*}, A_{i}^{*} i \mathrm{i}=1 \ldots 6$ are the flutter derivatives.

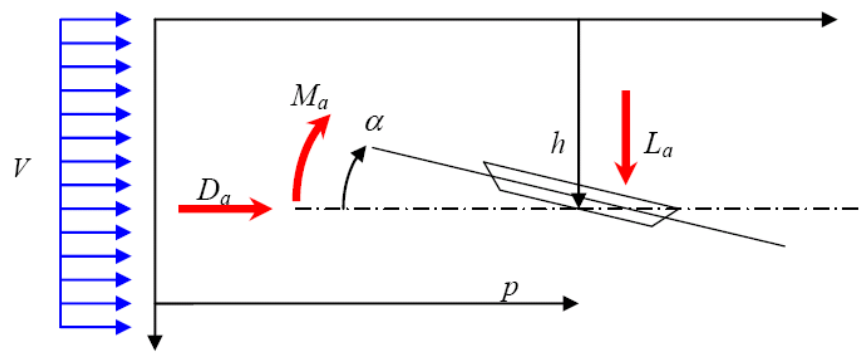

Figure 1: $\quad$ Sign criteria for the degree of freedom and the aeroelastic forces on the deck according to Scanlan formulation.

Because the flutter derivatives depend on $K$, it is usual to work in the frequency domain (Jurado et al. [2]). However, to work in the time domain it is necessary to express the aeroelastic forces as time functions, which is possible using the Wagner theory [3]. This aeronautic theory studies the displacements of a wing profile when a sudden change of the attack angle $\alpha_{0}$ takes place with respect to the position of no lift. The variation of lift with respect to the nondimensional time $(s=2 V t / B)$ is written by an indicial function $\Phi_{L, \alpha}(s)$ in the way 


$$
L_{\alpha}(s)=\frac{1}{2} \rho V^{2} B \hat{C}_{L} \alpha_{0} \Phi_{L \alpha}(s),
$$

where $\hat{C}_{L}$ is the derivative of the aerodynamic coefficient respect to the angle $\alpha$. The expression (3) can be generalized for any rotation by the Duhamel integral so

$$
L_{\alpha}(s)=\frac{1}{2} \rho V^{2} B \hat{C}_{L}\left[\Phi_{L \alpha}(0) \alpha(s)+\int_{0}^{s} \Phi_{L \alpha}^{\prime}(\sigma) \alpha(s-\sigma) d \sigma\right],
$$

where $\Phi_{L \alpha}^{\prime}$ is the derivative respect to $s$ of $\Phi_{L \alpha}$. In a similar way the expressions of the time variation of the lift caused by the vertical $\dot{h}$ and lateral $\dot{p}$ velocities of the system can be obtained. Therefore the total lift is

$$
\begin{aligned}
L_{a}(s)= & \frac{1}{2} \rho V^{2} B \hat{C}_{L}\left[\Phi_{L \alpha}(0) \alpha(s)+\int_{0}^{s} \Phi_{L \alpha}^{\prime}(\sigma) \alpha(s-\sigma) d \sigma\right]+ \\
& +\frac{1}{2} \rho V^{2} B \hat{C}_{L}\left[\Phi_{L h}(0) \frac{\dot{h}(s)}{V}+\int_{0}^{s} \Phi_{L h}^{\prime}(\sigma) \frac{\dot{h}(s-\sigma)}{V} d \sigma\right]+ \\
& -\frac{1}{2} \rho V^{2} B C_{L}\left[\Phi_{L p}(0) \frac{\dot{p}(s)}{V}+\int_{0}^{s} \Phi_{L p}^{\prime}(\sigma) \frac{\dot{p}(s-\sigma)}{V} d \sigma\right]
\end{aligned}
$$

and it depends on three indicial functions, one for each degree of freedom. The others aeroelastic forces drag, $D_{a}$ and moment $M_{a}$ have similar expressions:

$$
\begin{aligned}
D_{a}(s)= & \frac{1}{2} \rho V^{2} B \hat{C}_{D}\left[\Phi_{D \alpha}(0) \alpha(s)+\int_{0}^{s} \Phi_{D \alpha}^{\prime}(\sigma) \alpha(s-\sigma) d \sigma\right]+ \\
& +\frac{1}{2} \rho V^{2} B \hat{C}_{D}\left[\Phi_{D h}(0) \frac{\dot{h}(s)}{V}+\int_{0}^{s} \Phi_{D h}^{\prime}(\sigma) \frac{\dot{h}(s-\sigma)}{V} d \sigma\right]+ \\
& -\frac{1}{2} \rho V^{2} B C_{D}\left[\Phi_{D p}(0) \frac{\dot{p}(s)}{V}+\int_{0}^{s} \Phi_{D p}^{\prime}(\sigma) \frac{\dot{p}(s-\sigma)}{V} d \sigma\right] \\
M_{a}(s)= & \frac{1}{2} \rho V^{2} B \hat{C}_{M}\left[\Phi_{M \alpha}(0) \alpha(s)+\int_{0}^{s} \Phi_{M \alpha}^{\prime}(\sigma) \alpha(s-\sigma) d \sigma\right]+ \\
& +\frac{1}{2} \rho V^{2} B \hat{C}_{M}\left[\Phi_{M h}(0) \frac{\dot{h}(s)}{V}+\int_{0}^{s} \Phi_{M h}^{\prime}(\sigma) \frac{\dot{h}(s-\sigma)}{V} d \sigma\right]+ \\
& -\frac{1}{2} \rho V^{2} B C_{M}\left[\Phi_{M p}(0) \frac{\dot{p}(s)}{V}+\int_{0}^{s} \Phi_{M p}^{\prime}(\sigma) \frac{\dot{p}(s-\sigma)}{V} d \sigma\right]
\end{aligned}
$$

Indicial functions are not known and must be approximated by exponential functions fitting indices looking for the same force values that flutter derivatives produce. Another possibility is to use the Fourier transform of the indicial function expressed in frequency domain $\tilde{\Phi}^{\prime}(k)$, which has a direct relation with the flutter derivatives, as León et al. shows [4]. 


\section{Buffeting forces}

The dynamic equilibrium equation for the bridge deck includes the aeroelastic forces $\mathbf{f}_{a}$, analyzed in the previous paragraph, and the buffeting forces $\mathbf{f}_{b}$ caused by the turbulent nature of the wind.

$$
\mathbf{M u ̈}+\mathbf{C} \dot{\mathbf{u}}+\mathbf{K u}=\mathbf{f}_{a}+\mathbf{f}_{b},
$$

where $\mathbf{M}, \mathbf{C}$ and $\mathbf{K}$ are the mass, stiffness and damping matrices of the structure. The buffeting forces can also be written in a matrix form multiplying the matrix $\mathbf{P}_{b}$ by the wind fluctuations vector $\mathbf{w}_{b}$, in the wind direction $v_{v}$ and vertical $w_{v}$. The matrix $\mathbf{P}_{b}$ contains the aerodynamic coefficients of drag $C_{D}$, lift $C_{L}$, and moment $C_{M}$, its derivatives with respect to the angle of attack $C^{\prime}{ }_{D}, C^{\prime}{ }_{L}$ and $C^{\prime}{ }_{M}$ and the admittance functions $\chi_{D u v}, \chi_{L u v}, \chi_{M u v}, \chi_{D w v}, \chi_{L w v}, \chi_{M w v}$ which take into account the dependency of the aerodynamic coefficient of the frequency.

$$
\mathbf{f}_{a}=\mathbf{P}_{b} \mathbf{w}_{b}=\frac{1}{2} \rho V^{2} B l\left(\begin{array}{cc}
\frac{2 C_{D}}{V} \chi_{D u_{v}} & \frac{C^{\prime}{ }_{D}}{V} \chi_{D w_{v}} \\
\frac{2 C_{L}}{V} \chi_{L u_{v}} & \frac{\left(C_{L}^{\prime}+C_{D}\right)}{V} \chi_{L w_{v}} \\
B \frac{2 C_{M}}{V} \chi_{M u_{v}} & B \frac{C_{M}^{\prime}}{V} \chi_{M w_{v}}
\end{array}\right)\left\{\begin{array}{c}
v_{v}(t) \\
w_{v}(t)
\end{array}\right\} .
$$

The buffeting phenomenon is usually analyzed in the frequency domain with spectral analysis. In fact, the wind turbulence is defined by the spectrum of the wind speed fluctuations. Before a time domain analysis, it is necessary to generate a time history of the wind speed from the known fluctuation spectrums. The Shinozuka and Deodatis method [5] is based on a sinus expansion and an inverse Fourier transform can be used for that purpose. For example, the time history of figure 2 is generated for a mean wind speed of $V=4 \mathrm{~m} / \mathrm{s}$ and a vertical fluctuation spectrum $S_{w}$ given by

$$
\begin{gathered}
S_{w}(z, f)=\frac{6.103 L_{w} I_{w}^{2} V(z)}{\left[1+63.181\left(f L_{w} / V(z)\right)\right]} \\
L_{w}=0.10 L_{u}, L_{v}=300(z / 200)^{1 / 2}, I_{w}=0.5 I_{u}, I_{v}=1 / \ln \left(z / z_{0}\right), z_{0}=0.05 \mathrm{~m}
\end{gathered}
$$

which depends on turbulence scales $L$, turbulence intensities $I$ and the terrain roughness $z_{0}$.

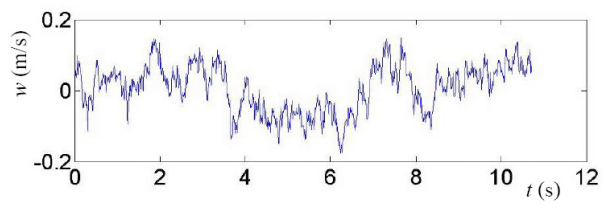

Figure 2: Time history of the vertical wind fluctuation. 


\section{Quasi-steady approach (QS)}

The quasi-steady theory is based on the hypothesis that wind forces are stationary. An apparent wind speed considering the deck movement is defined, so the expressions of wind forces are the usual static functions with the aerodynamic coefficients. This hypothesis is valid when the vibration frequency of the deck is low under high wind speed, so the deck movement does not affect the flow around itself. If the turbulent forces are taken into account, the theory is only right when eddies have low frequency and great scale. Small eddies with high frequency affect only a part of the deck and therefore this theory is not applicable.

The aerodynamic coefficients depend on the angle of attack between the deck and the instantaneous flow. This angle is usually call dynamic attack angle $\alpha_{d}$. Figure 3 shows this angle defined as the sum of static deck rotation $\theta_{s t}$, the angle caused by the instantaneous rotational vibration $\theta$ and the angle $\psi$ which takes into account the relative velocity $V_{r}$ between the turbulent flow with fluctuations $v_{v}$ and $w_{v}$, and the deck velocities $\dot{\mathbf{u}}=\{\dot{y}, \dot{z}, \dot{\theta}\}$. The wind forces expressions are then

$$
F_{y^{\prime}}=\frac{1}{2} \rho V_{r y}^{2} B C_{D}\left(\alpha_{d}\right) ; F_{y^{\prime}}=\frac{1}{2} \rho V_{r z}^{2} B C_{L}\left(\alpha_{d}\right) ; F_{\theta}=\frac{1}{2} \rho V_{r \theta}^{2} B C_{M}\left(\alpha_{d}\right),
$$

where

$$
V_{r i}^{2}=\left(V+v_{v}-\dot{y}\right)^{2}+\left(w_{v}-B_{1, i} \dot{\theta}-\dot{z}\right)^{2} .
$$

$B_{1, i}$ is a correction coefficient for the deck width used to consider the distance between the rotation centre and the geometrical centre of the deck. Rocchi [6] gives an expression to obtain this using the flutter derivatives (Polytechnic of Milan criteria)

$$
B_{1, y}=B \frac{p_{2}^{*}}{p_{1}^{*}} ; B_{1, z}=B \frac{h_{2}^{*}}{h_{1}^{*}} ; B_{1, \theta}=B \frac{a_{2}^{*}}{a_{1}^{*}} .
$$

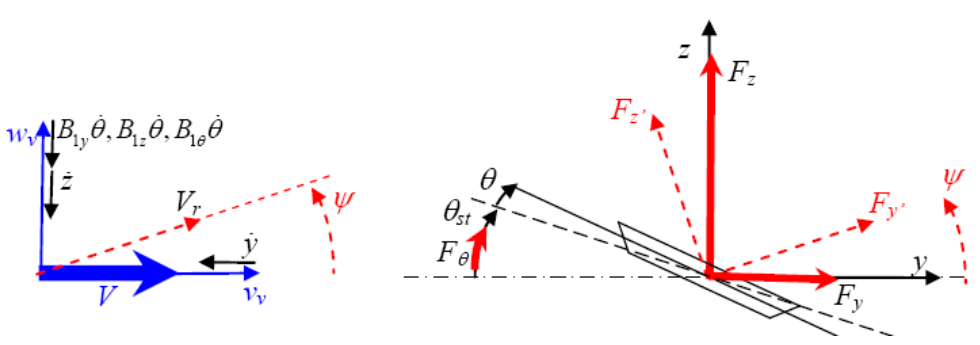

Figure 3: $\quad$ Dynamic angle of attack.

As shown in figure 3, the forces have the apparent wind directions and a projection on the global axis is necessary to calculate the quasi-steady forces vector $\mathbf{f}_{s}$. After that, the modal analysis is used to reduce the dimension problem and, finally, a step by step direct integration permits the resolution of the displacement equations: for example using the Wilson- $\theta$ method that is very well described in many texts as Clough and Penzien [7]. 


$$
\begin{aligned}
& \mathbf{M} \ddot{\mathbf{u}}+\mathbf{C} \dot{\mathbf{u}}+\mathbf{K u}=\mathbf{f}_{s} ; \quad \mathbf{u}=\boldsymbol{\Phi} \mathbf{q} \\
& \mathbf{I} \ddot{\mathbf{q}}+\boldsymbol{\Phi}^{T} \mathbf{C} \boldsymbol{\Phi} \dot{\mathbf{q}}+\boldsymbol{\Phi}^{T} \mathbf{K} \boldsymbol{\Phi} \mathbf{q}=\boldsymbol{\Phi}^{T} \mathbf{f}_{s}
\end{aligned}
$$

The quasi-steady approach has advantages to permit a non-linear analysis considering the variation of the angle of attack caused by the torsional rotation of the deck. The instantaneous bridge deformation changes the angle of attack in respect to a previous time affecting the wind forces definition. This non-linearity cannot be analysed by the methods that use flutter derivatives or indicial functions because they fix the angle of attack around which the vibrations take place. Besides, they consider small displacements.

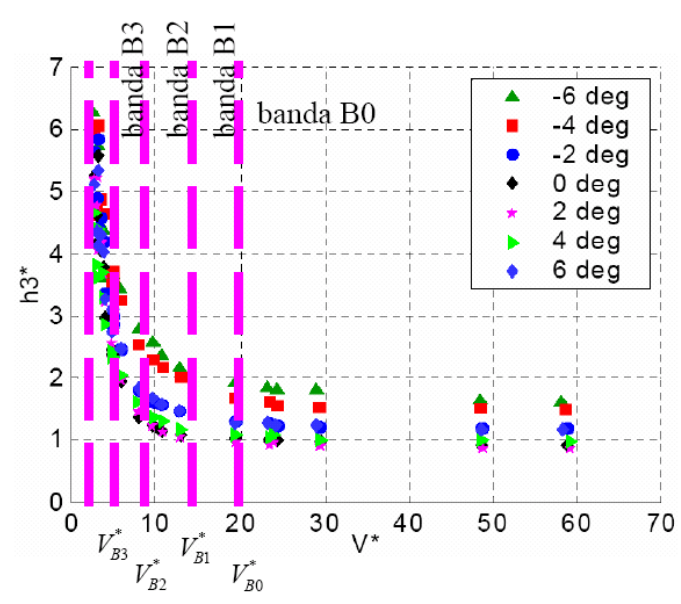

Figure 4: $\quad$ Band divisions depending on the flutter derivative value $h_{3}^{*}$ (Milan criteria).

\section{Band superposition method (BS)}

The band superposition method has been recently developed by Diana et al. [8] and Rocchi [6], professors of the Polytechnic of Milan. The new method combines the easy definition of wind forces as frequency functions, such as the flutter derivatives, and the possibility of a non-linear analysis, which is a characteristic of the quasi-steady approach. Several sets of wind forces depending on the frequency must be established. The total range of frequencies is divided by bands. For the low frequencies, band B0, the quasi-steady approach is used and the structural response is obtained in time domain, as explained in the previous section. Then, using the resulted dynamic angle of attack of the band B0, the flutter derivatives and the aerodynamic coefficients are evaluated for the upper bands, using frequency functions for the wind forces. The total displacements solution is the sum of all the bands responses. There are several possibilities to choose the frequency of each upper band: the mean frequency of the band (BSWbandQ), or the damping frequency of each aeroelastic mode (BSWdaQ). 
The number of bands and their frequency limits are determined by the values of the flutter derivatives, considering the Milan criteria (figure 4). According to this criteria some flutter derivatives have an asymptote for high values of the reduced velocity $V^{*}=2 \pi V / \omega B$, which corresponds to low frequencies (band B0). For many deck section shapes, the lower limit of reduced velocity is $V_{B 0}^{*}=20$. To determine the rest of the band limits, the maximum variation of the flutter derivative values is fixed. The cut frequency for one band is $\omega_{B l}=2 \pi V /\left(B V_{B l}^{*}\right)$. In each band the frequency functions are evaluated with the mean value of the upper and lower frequency limits $\bar{\omega}_{B j}=\left(\omega_{B j}+\omega_{B j-1}\right) / 2$. A time history of wind fluctuations $\mathbf{w}_{b}(t)$ is evaluated by the Shinozuka-Deodatis method, yet using only the frequencies of the band. After that, the buffeting forces vector is calculated by expression (9). The method analyzes the band B0 by the quasi-steady approach expressed in (14), and each upper band $j$ by step by step direct integration after a modal decomposition, solving

$$
\mathbf{M} \ddot{\mathbf{u}}_{B j}+\mathbf{C} \dot{\mathbf{u}}_{B j}+\mathbf{K} \mathbf{u}_{B j}=\mathbf{f}_{a, B j}+\mathbf{f}_{b, B j} ; \quad \mathbf{u}_{B j}=\boldsymbol{\Phi} \mathbf{q}_{B j} .
$$

The BSWbandQ method needs to build different aeroelastic matrices in each band and takes the band mean frequency, which it is expressed by

$$
\mathbf{I} \ddot{\mathbf{q}}_{B j}+\boldsymbol{\Phi}^{T}\left[\mathbf{C}-\mathbf{C}_{a}\left(\alpha_{d}, \bar{\omega}_{B j}\right)\right] \boldsymbol{\Phi} \dot{\mathbf{q}}_{B j}+\boldsymbol{\Phi}^{T}\left[\mathbf{K}-\mathbf{K}_{a}\left(\alpha_{d}, \bar{\omega}_{B j}\right)\right] \boldsymbol{\Phi} \mathbf{q}_{B j}=\boldsymbol{\Phi}^{T} \mathbf{f}_{b}\left(\alpha_{d}, \bar{\omega}_{B j}\right) .
$$

However, the BSWdaQ method uses the same aeroelastic matrices for all the bands and builds them with the damping frequency $\omega_{d, j}$ of each aeroelastic mode. In this case the equations system is

$$
\begin{aligned}
& \bar{C}_{a, i j}=\boldsymbol{\phi}_{i}^{T}\left[C_{a, i j}\left(\alpha_{d}, \omega_{d, j}\right)\right] \phi_{j} ; \quad \bar{K}_{a, i j}=\boldsymbol{\phi}_{i}^{T}\left[K_{a, i j}\left(\alpha_{d}, \omega_{d, j}\right)\right] \phi_{j} \\
& \mathbf{I}_{\mathbf{q}_{B j}}+\left[\boldsymbol{\Phi}^{T} \mathbf{C} \boldsymbol{\Phi}-\overline{\mathbf{C}}_{a}\right] \dot{\mathbf{q}}_{B j}+\left[\boldsymbol{\Phi}^{T} \mathbf{K} \boldsymbol{\Phi}-\overline{\mathbf{K}}_{a}\right] \mathbf{q}_{B j}=\boldsymbol{\Phi}^{T} \mathbf{f}_{b}\left(\alpha_{d}, \bar{\omega}_{B j}\right)
\end{aligned} .
$$

The total displacements in a time $t$ are approximated by the sum of the band responses for that time

$$
\mathbf{u}(t)=\sum_{B j=B 0}^{B n} \mathbf{u}_{B j}(t) ; \quad \dot{\mathbf{u}}(t)=\sum_{B j=B 0}^{B n} \dot{\mathbf{u}}_{B j}(t) ; \quad \ddot{\mathbf{u}}(t)=\sum_{B j=B 0}^{B n} \ddot{\mathbf{u}}_{B j}(t) .
$$

\section{Inverse Fourier transform of the frequency response (IFTR)}

A time history of the deck displacements can be easily obtained by an inverse Fourier transform (IFRT) of the frequency domain response. This approach is an option to check the previous methods, although it also assumes the hypothesis of small displacement amplitudes around the initial position. Starting from the wind velocity fluctuations vector expressed in time domain $\mathbf{w}_{b}(t)=\left[v_{v}(t), w_{v}(t)\right]^{T}$, the Fourier transform changes it to frequency domain $\tilde{\mathbf{w}}_{b}(\omega)$. After that, the buffeting forces are calculated by $\tilde{\mathbf{f}}_{b}(\omega)=\mathbf{P}_{b} \tilde{\mathbf{w}}_{b}(\omega)$. Using modal analysis the participation modal vector can be written in the following form,

$$
\mathbf{u}=\boldsymbol{\Phi q} ; \quad \mathbf{q}(t)=\mathbf{q}_{0} e^{i \omega t} ; \tilde{\mathbf{q}}(\omega)=\int_{-\infty}^{\infty} \mathbf{q}(t) e^{-i \omega t} d t ; \quad \tilde{\mathbf{u}}=\boldsymbol{\Phi} \tilde{\mathbf{q}},
$$

the equations system ( 8 ) becomes 


$$
\begin{gathered}
\left\{\left[\boldsymbol{\Phi}^{T}\left(\mathbf{K}-\mathbf{K}_{a}(\omega)\right) \boldsymbol{\Phi}-\omega^{2} \mathbf{I}\right]+i \omega \boldsymbol{\Phi}^{T}\left(\mathbf{C}-\mathbf{C}_{a}(\omega)\right) \boldsymbol{\Phi}\right\} \tilde{\mathbf{q}}(\omega)=\Phi^{T} \tilde{\mathbf{f}}_{b}(\omega) \\
\mathbf{V}(\omega) \tilde{\mathbf{q}}(\omega)=\Phi^{T} \tilde{\mathbf{f}}_{b}(\omega) \Rightarrow \tilde{\mathbf{q}}(\omega)=\mathbf{V}^{-1}(\omega) \Phi^{T} \tilde{\mathbf{f}}_{b}(\omega) \Rightarrow \tilde{\mathbf{q}}(\omega)=\mathbf{H}(\omega) \Phi^{T} \tilde{\mathbf{f}}_{b}(\omega)
\end{gathered}
$$

where $\mathbf{V}(\omega)$ is the impedance matrix and its inverse is the transfer matrix $\mathbf{H}(\omega)$. The IFRT of $\tilde{\mathbf{u}}(\omega)$ finally gives $\mathbf{u}(t)$. The solution is a periodic function, so it is a stationary response. Therefore IFRT does not permit one to analyse a nonstationary instability as flutter, because in this phenomena the displacements amplitudes increase and are not periodic.

\section{Examples}

The first example consists of a flat plate section of $B=1.2 \mathrm{~m}$ width with only one degree of freedom of torsional rotation $\varphi$. The following properties are taken into account per unit length: torsional inertia $I=1 \mathrm{kgm}^{2}$, damping $c=0.2$ $\mathrm{kg} \cdot \mathrm{s} / \mathrm{m}^{2}$, stiffness $k=25.266 \mathrm{~kg} \cdot \mathrm{s}^{2} / \mathrm{m}^{2}$, the natural frequency is $\omega=(k / I)^{1 / 2}=$ $5.026 \mathrm{rad} / \mathrm{s}$ and the damping ratio is $\xi=c / 2 I \omega=0.02$. If a wind speed $V=4 \mathrm{~m} / \mathrm{s}$ is considered and taking the fluctuations shown in figure 2 , the following wind forces are evaluated:

- Aeroelastic moment from expression (1) and (2) using the flutter derivatives of a flat plate (Simiu and Scanlan [1]) and taking the natural frequency $\omega_{n}$, the aeroelastic damping frequency $\omega_{d a}$, or the bands frequencies $\omega_{\text {band }}$.

- Buffeting moment from the expression (9) $M_{b}=1 / 2 \rho V^{2} B^{2} C^{\prime}{ }_{M}\left(w_{v} / V\right)$.

The displacements equation of the system $I \ddot{\varphi}+c \dot{\varphi}+k \varphi=M_{a}+M_{b}$ has been solved by the following methods:

- IFTR: Inverse Fourier transform of the frequency response.

..... Wilson $\theta$ with indicial functions related with flutter derivatives $A_{2}^{*} \mathrm{y} A_{3}^{*}$.

----- BSWn: Band superposition method without QS for band B0 and using the natural frequency to calculate $A_{2}^{*}$ y $A_{3}^{*}$.

BSWda: Band superposition method without QS for band B0 and using the aeroelastic damping frequency to calculate $A_{2}^{*}$ y $A_{3}^{*}$.

BSWband: Band superposition method without QS for band B0 and using the band frequencies to calculate $A_{2}^{*} \mathrm{y} A_{3}^{*}$.

Figure 5 shows the time histories of the flat plate rotation in each case. The Wilson $\theta$ solution with indicial functions perfectly fits to IFRT because both methods suppose small displacements and the indicial functions are evaluated from the flutter derivatives. The BSWband solution fits to IFRT during the stationary part but not at the beginning. This happens because the flutter derivatives are calculated with the band frequencies and not using the response frequency. BSWn and BSWda solutions present the worst results.

The main aim of this paper is to obtain the wind response of a long-span bridge in the time domain, so the Akashi Bridge in Japan, record of span length (1991 m), has been taken as an example. Its structural and aerodynamic properties and the wind characteristics, profile and fluctuation spectrums can be found in Katsuchi [10]. The time history of wind velocity has been calculated 

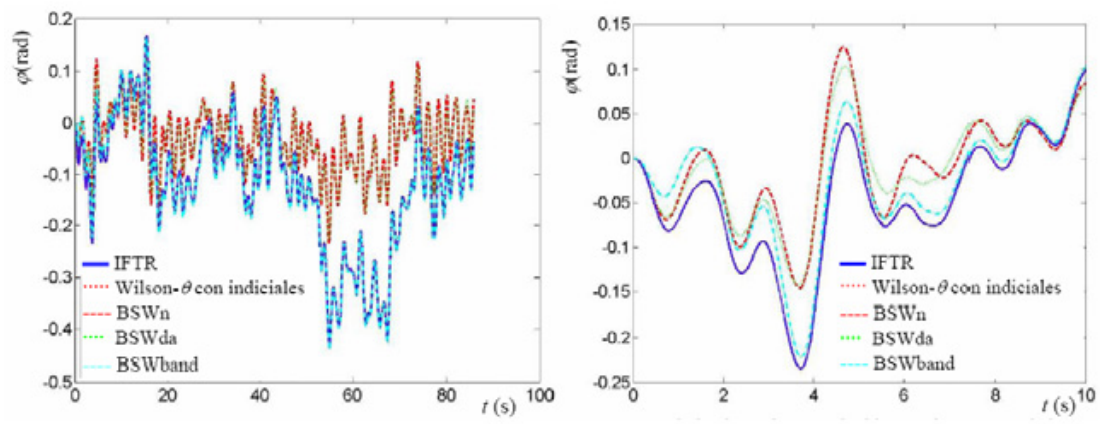

Figure 5: Time histories of the flat plate rotation.
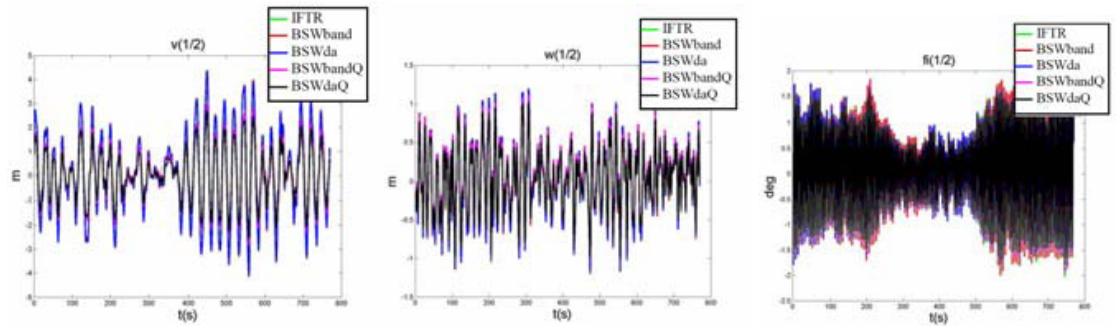

Figure 6: $\quad$ Lateral $v$, vertical $w$ and rotational $\varphi$ displacements time histories of the central point.

during 13 minutes using the Shinozuka-Deodatis method from the fluctuation spectrums. A mean wind speed of $60 \mathrm{~m} / \mathrm{s}$ is considered. The deck displacements response is shown in figure 6 using the following approaches:

IFRT: Inverse Fourier transform of the frequency response.

- BSWband: Band superposition method without QS for band B0 and using the band frequencies.

- BSWda: Band superposition method without QS for band B0 and using the aeroelastic damping frequencies.

- BSWbandQ: Band superposition method using QS for band B0 and the band frequencies.

- BSWdaQ: Band superposition method using QS for band B0 and the aeroelastic damping frequencies.

To study the displacement graphs of figure 6 with all the curves together is difficult, so the root mean square of the degrees of freedom along the bridge spans has been calculated from the time histories. The three first methods more or less show the same results, which are also similar to Katsuchi's [10] solution. The two methods that use the quasi-steady approach to band B0 give substantially different results, mainly for the lateral displacement which is $40 \%$ smaller. It is curious that testing in the wind tunnel of the Akashi bridge full model also gave smaller lateral vibrations, this confirms that methods based on band superposition using quasi-steady solution for band B0 are the best. 

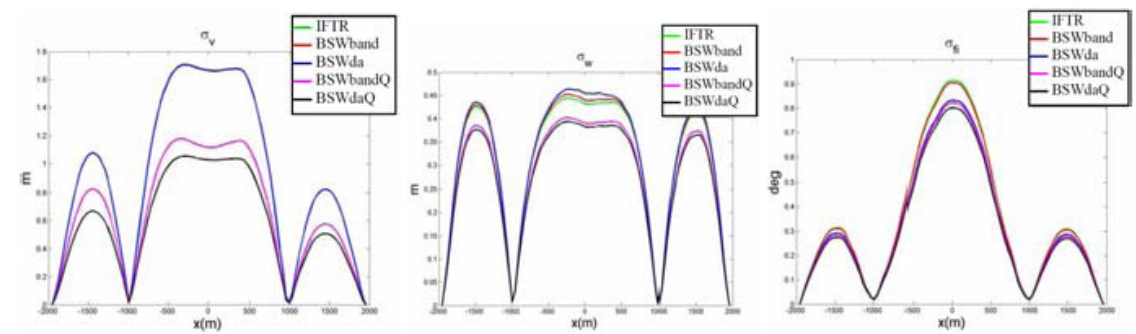

Figure 7: Lateral $v$, vertical $w$ and rotational $\varphi$ root mean square displacements along the spans.

\section{Conclusions}

- The current calculation power of computers permits the aeroelastic analysis of long-span bridges in the time domain.

- Working in the time domain permits one to more accurately study non-stationary periods associated with the initiation of the aeroelastic instabilities, and also nonlinearities can be analyzed more easily.

- The quasi-steady approach has advantages to permit a non-linear analysis considering the variation of the angle of attack caused by the torsional rotation of the deck.

- A time history of the deck displacements can be obtained by an inverse Fourier transform (IFRT) of the frequency domain response. This approach is an option to check the previous methods, although it also assumes the hypothesis of small displacement amplitudes around the initial position.

- The Wilson $\theta$ solution with indicial functions perfectly fits to IFRT because both methods suppose small displacements and the indicial functions are evaluated from the flutter derivatives.

- Band superposition consists of a decomposition of the wind forces in different frequency ranges, analyzing the forces of the lowest frequency band as quasi-steady, and the higher frequency bands with their classical expressions in terms of frequency.

- The methods that use band superposition and the quasi-steady approach to band $\mathrm{B} 0$ give better results, according to the testing of the Akashi Bridge full model in a wind tunnel.

\section{References}

[1] Simiu E. and Scanlan R. H.; "Wind Effects on Structures”, Fundamentals and Applications to Design. John Wiley \& Sons, 1996.

[2] Jurado J. A. León A. Nieto F. y Hernandez S. "FLAS-B: Software for the Hybrid Analysis of Flutter and Buffeting Phenomena". $4^{\circ}$ International Symposium on Computation Wind Engineering. Yocohama Japón. 16-19 
Julio 2006. Journal of Wind Engineering, JAWE Vol. 31 No. 108, pag. 323-326.

[3] Wagner H. "Uber die Entstehung des dynamischen Auftriebes von Tragflügeln”. Z., Angew. Math. Mech Bd. 5-Helft 1. 1925.

[4] León A. Jurado, J. A. Hernandez S. y Nieto F. "Comparación de diversas funciones indiciales para la resolución del flameo de una placa plana en el dominio del tiempo". Congreso de Métodos Numéricos en Ingeniería 2007. Oporto Portugal, 13- 15 junio.

[5] Shinozuka, M. and Deodatis G. "Simulation of stochastic processes by spectral representation”. Applied Mechanics 44 (4): 191-204. 1991.

[6] Rocchi D. "Effetti non lineari nella risposta areoelastica di ponti sospesi a grande luce". dottorato di ricerca in meccanica applicata. Tesi di dottorato Politecnico de Milano, 2004.

[7] Clough R. W., Penzien J. "Dynamics of Structures". 2nd Edition. McGrawHill. New York. 1993.

[8] Diana G. Resta F. Rocchi D. "A New Numerical Approach to Reproduce Bridge Aerodynamic Non Linearity in Time Domain" 16-19 Julio 2006. $4^{\circ}$ International Symposium on Computational Wind Engineering. Yokohama, Journal of Wind Engineering, JAWE Vol. 31 No. 108.

[9] Jurado J. A. Hernández S. "Sensitivity analysis of bridge flutter speed with respect to mechanical parameters of the deck". Structural and multidisciplinary optimization, Vol. 27, pp. 272-283. 2004

[10] Katsuchi, H. "An Analytical Study on Flutter and Buffeting of The AkashiKaikyo Bridge". An essay submitted to The Johns Hopkins University in conformity with the requirements for the degree of Master of Science in Engineering. Baltimore, Maryland. 1997. 